\title{
A Promoter Polymorphism of Lamin A/C Gene is an Independent Genetic Predisposition to Arterial Stiffness in a Japanese General Population (The Tanno and Sobetsu Study)
}

\author{
Hiroshi Akasaka ${ }^{1}$, Tomohiro Katsuya ${ }^{2}$, Shigeyuki Saitoh ${ }^{1}$, Ken Sugimoto ${ }^{2}$, Hirofumi Ohnishi ${ }^{1}$, \\ Ada Congrains ${ }^{2}$, Miyuki Ohnishi ${ }^{2}$, Mitsuru Ohishi², Hiromi Rakugi ${ }^{2}$, Toshio Ogihara ${ }^{2,3}$, \\ and Kazuaki Shimamoto ${ }^{1}$
}

\footnotetext{
${ }^{1}$ Second Department of Internal Medicine, Sapporo Medical University School of Medicine, Hokkaido, Japan

${ }^{2}$ Department of Geriatric Medicine, Osaka University Graduate School of Medicine, Osaka, Japan

${ }^{3}$ Osaka General Medical Center, Osaka Prefectural Hospital Organization, Osaka, Japan
}

\begin{abstract}
Aim: We examined the hypothesis that there is a positive, independent association between polymorphisms of lamin A/C gene (LMNA) and arterial stiffness in Japanese.

Methods: The subjects were 261 men (mean age, 64.4 \pm 0.7 years) selected from inhabitants of the towns of Tanno and Sobetsu in a rural area of Japan who underwent medical check-ups. We conducted clinical examinations, including measurement of bilateral brachial-ankle pulse wave velocity (baPWV) as a marker of arterial stiffness, and genetic analysis. Subjects with atrial fibrillation, subjects with ankle-brachial index $<0.9$, and subjects taking any medication were excluded. We selected two single nucleotide polymorphisms (SNPs) as markers of $L M N A, 1908 \mathrm{C} / \mathrm{T}$ in exon 10 and $-1030 \mathrm{C} / \mathrm{T}$ in the promoter region, which we have recently identified. All genotypes were clearly determined by the TaqMan PCR method.

Results: Genotype frequencies of the two polymorphisms satisfied the Hardy-Weinberg equilibrium. The baPWV of $-1030 \mathrm{C} / \mathrm{T}$ polymorphism was significantly greater in subjects with CC genotype than in subjects with CT + TT genotype $(1,652 \pm 22.1 \mathrm{~cm} / \mathrm{s}$ vs. $1,552 \pm 43.0 \mathrm{~cm} / \mathrm{s}, p=0.039)$; however, no significant difference was found for $1908 \mathrm{C} / \mathrm{T}$ polymorphism. The baPWV was found to be significantly associated with age, body height, systolic blood pressure, and smoking habit; therefore, we next performed multiple regression analysis including these parameters, and found an independent, significant association between baPWV and $-1030 \mathrm{C} / \mathrm{T}$ polymorphism.

Conclusion: Promoter $-1030 \mathrm{C} / \mathrm{T}$ polymorphism of $L M N A$ is a possible genetic predisposition to arterial stiffness in the Japanese population.
\end{abstract}

J Atheroscler Thromb, 2009; 16:404-409.

Key words; Arterial stiffness, Nuclear lamina, Genetics, Single nucleotide polymorphism (SNP)

\section{Introduction}

Hutchinson-Gilford progeria syndrome (HGPS; Online Mendelian Inheritance in Man \#176670) is a rare sporadic disorder with premature aging, and

Address for correspondence: Hiroshi Akasaka, Second Department of Internal Medicine, Sapporo Medical University School of Medicine, Sapporo, Hokkaido 060-8543, Japan

E-mail: akasaka@sapmed.ac.jp

Received: December 17, 2008

Accepted for publication: January 29, 2009 patients with this syndrome are likely to have coronary artery disease, stroke, or other cardiovascular diseases $^{1-3)}$. HGPS induces severe systemic arterial stiffness, which leads to fatal myocardial infarction or stroke before an average age of 13 years. Approximately $80 \%$ of HGPS cases are caused by a single base change of $\mathrm{C}$ to $\mathrm{T}$ in position 1824 on exon 11 of a gene encoding nuclear lamins $\mathrm{A}$ and $\mathrm{C}^{4,5)}$. Lamins are structural protein components of nuclear lamina, a protein network underlying the inner nuclear membrane that determines nuclear shape and size, and 
constitute a class of intermediate filaments. The gene encoding lamins $\mathrm{A}$ and $\mathrm{C}$ is named lamin $\mathrm{A} / \mathrm{C}$ gene ( $L M N A$; Gene ID 4000), and it spans approximately $24 \mathrm{~kb}$ and contains 12 exons on chromosome 1q21. Alternative splicing within exon 10 of $L M N A$ gives rise to 2 different mRNAs that code for prelamin A and lamin C.

HGPS is associated with premature arterial stiffness, and it is therefore thought that LMNA is involved in the pathophysiology and genesis of arterial stiffness that occurs concurrently with the accelerated aging process. It has been reported that LMNA has several single nucleotide polymorphisms (SNPs). The $1908 \mathrm{C} / \mathrm{T}$ polymorphism (rs \#4641), one of the SNPs on $L M N A$, in exon 10 is associated with a risk for developing metabolic traits, including insulin resistance. A positive association between $1908 \mathrm{C} / \mathrm{T}$ polymorphism and metabolic abnormalities has been reported in Inuit $^{6)}$, Japanese ${ }^{7)}$, Pima Indians and Armish ${ }^{8)}$.

However, there is no data on the association between $L M N A$ and arterial stiffness in a general population. Arterial stiffness is mainly determined by measuring pulse wave velocity (PWV) ${ }^{9}$. PWV reflects systemic arteriosclerosis as well as relating to cardiovascular risk factors ${ }^{10)}$ and ischemic heart disease in type 2 diabetes mellitus ${ }^{11}$. PWV is also a predictor of cardiovascular mortality in patients with end-stage renal disease ${ }^{12)}$ or hypertension ${ }^{13)}$ and in elderly individuals $^{14)}$, independently of age, blood pressure, and cardiac mass.

The purpose of this study was to examine the relationship between polymorphisms of $L M N A$ and arterial stiffness in a cross-sectional epidemiological study of a Japanese general population, the Tanno and Sobetsu study.

\section{Materials and Methods}

We recruited 586 male inhabitants of Tanno Town and Sobetsu Town who had undergone medical checkups in 2003. Tanno and Sobetsu are located in Hokkaido, the northernmost island of Japan. The Tanno and Sobetsu study was started in 1977 with a population-based prospective cohort design. Detailed epidemiological findings have already been reported ${ }^{15-18)}$.

The subjects completed a standard questionnaire regarding their medical history and their smoking and drinking habits. We measured anthropometric parameters, systolic blood pressure (SBP), diastolic blood pressure (DBP), total cholesterol, triglyceride, highdensity lipoprotein (HDL) cholesterol, plasma glucose, immunoreactive insulin (IRI), highly sensitive
C-reactive protein (hs-CRP), and adiponectin in all subjects. Brachial-ankle pulse wave velocity (baPWV) and ankle-brachial index (ABI) were measured using Form ${ }^{\circledR}$ PWV/ABI (Omron Colin Co., Ltd., Tokyo, Japan) and the average of right baPWV and left baPWV was adopted ${ }^{19}$. Insulin sensitivity was determined by homeostasis model assessment of the insulin resistance (HOMA-IR) index, which was calculated as plasma glucose $(\mathrm{mg} / \mathrm{dL}) \times$ immunoreactive insulin $(\mu \mathrm{U} / \mathrm{L}) / 405$. Blood samples were collected in the early morning after fasting for 8-11 hours. Blood pressure was measured twice after 5 minutes of rest, with the subjects seated.

Exclusion criteria were atrial fibrillation, suspected arteriosclerosis obliterans (ASO) defined as ABI on any side lower than 0.9 , and taking any medication, in order to rule out drug effects. After excluding 219 of the 586 male subjects according to the above criteria, we conducted genetic analysis. Finally, 261 male subjects were successfully genotyped. All subjects gave written informed consent to participate in the genetic analysis and in all other procedures associated with the study. The Institutional Review Board (IRB) of Osaka University and the IRB of Sapporo Medical University both approved the study protocol.

Genomic DNA was extracted from $200 \mu \mathrm{L}$ buffy coat using a QIAamp DNA Blood Kit (QIAGEN K. K., Tokyo, Japan). C-to-T transversion at nucleotide position 1908 in exon 10 of the lamin $\mathrm{A} / \mathrm{C}$ gene (LMNA 1908C/T; rs \#4641) and C-to-T transversion at nucleotide position -1030 in the promoter region of LMNA (LMNA -1030C/T; no rs\#) were determined by the TaqMan-polymerase chain reaction (PCR) method. The LMNA 1908C/T polymorphism was detected using the following primers and probes: forward, 5'-CGA GGA TGA GGA TGG AGA TGA C-3'; reverse, 5'-CCT CAG CGG CGG CTA C-3'; cytosine base (C)-specific probe, 5'-VIC-CAC TCA CGT GGT GGT G-MGB-3'; and thymine base (T)specific probe, 5'-FAM-CAC TCA CAT GGT GGT G-MGB-3'. The $L M N A-1030 \mathrm{C} / \mathrm{T}$ polymorphism was detected using the following primers and probes: forward, 5'-CCA CTA CCT TCT TTC TGG CTG AA-3'; reverse, 5'-ACT AGG TCC CAG ATT TCT GTG GTT-3'; cytosine base (C)-specific probe, 5'VIC-CAG CCA ATG TTG GGT C-MGB-3'; and thymine base (T)-specific probe, 5'-FAM-ACA GCC AAT ATT GGG TC-MGB-3'. PCR was carried out using a GeneAmp PCR System 9700 thermal cycler (Applied Biosystems, Foster City, CA, USA). PCR conditions were as follows: initial denaturation at $95^{\circ} \mathrm{C}$ for $10 \mathrm{~min}$, followed by 40 cycles of $92^{\circ} \mathrm{C}$ for 15 sec and $60^{\circ} \mathrm{C}$ for $60 \mathrm{sec}$. The fluorescence level of PCR 
Table 1. Baseline characteristics of study subjects $(n=261)$

\begin{tabular}{lc}
\hline & Male $(n=261)$ \\
\hline Age (years) & $64.6 \pm 0.7$ \\
BMI $\left(\mathrm{kg} / \mathrm{m}^{2}\right)$ & $23.5 \pm 0.2$ \\
SBP $(\mathrm{mmHg})$ & $133 \pm 1.3$ \\
DBP $(\mathrm{mmHg})$ & $75 \pm 0.7$ \\
Total cholesterol $(\mathrm{mg} / \mathrm{dL})$ & $193 \pm 2.0$ \\
Triglyceride $(\mathrm{mg} / \mathrm{dL})$ & $112 \pm 4.6$ \\
HDL cholesterol $(\mathrm{mg} / \mathrm{dL})$ & $52 \pm 0.8$ \\
Current smoker $(\%)$ & 33.0 \\
HOMA-IR & $1.0 \pm 0.07$ \\
hsCRP $(\mathrm{mg} / \mathrm{dL})$ & $0.107 \pm 0.008$ \\
Adiponectin $(\mathrm{ng} / \mathrm{mL})$ & $6.1 \pm 0.2$ \\
baPWV $(\mathrm{cm} / \mathrm{s})$ & $1,631 \pm 19.7$ \\
\hline
\end{tabular}

Values are expressed as the mean \pm SEM or \%. BMI, body mass index; SBP, systolic blood pressure; DBP, diastolic blood pressure; HDL, high-density lipoprotein; HOMA-IR, homeostasis assessment model of insulin resistance; hsCRP, highly sensitive C-reactive protein; baPWV, brachial-ankle pulse wave velocity

products measured using an ABI PRISM 7900HT Sequence Detector (Applied Biosystems) differentiated the three genotypes of these two polymorphisms.

Associations between the polymorphisms and clinical variables were analyzed using one-way analysis of variance (ANOVA). Differences in genotype or allele distribution were examined by $\chi^{2}$ analysis. Multiple regression analysis was used to assess the contribution of confounding factors. All numerical values are expressed as the means \pm SEM. Significance was defined as $p<0.05$. All statistical analyses were conducted using JMP software version 5.1.2J for Windows (SAS Institute Inc., Cary, NC, USA).

\section{Results}

The 261 male subjects had a mean age of $64.6 \pm$ 0.7 years, mean body mass index (BMI) of $23.5 \pm 0.2$ $\mathrm{kg} / \mathrm{m}^{2}$, and mean brachial-ankle pulse wave velocity (baPWV) of 1,631 $\pm 19.7 \mathrm{~cm} / \mathrm{sec}$. Table 1 shows the baseline characteristics of all study subjects. The genotype frequencies of the two polymorphisms of $L M N A$ examined did not significantly differ from the values predicted by the Hardy-Weinberg equilibrium. The frequencies of CC, CT and TT genotypes of exon 10 $1908 \mathrm{C} / \mathrm{T}$ polymorphism were $60 \%, 32 \%$ and $8 \%$, respectively, and the frequencies of $\mathrm{CC}$, CT and TT genotypes of promoter $-1030 \mathrm{C} / \mathrm{T}$ polymorphism were $79 \%, 16 \%$ and $5 \%$, respectively. Since the number of subjects with TT genotype of these two polymorphisms was small, we adopted a recessive model of the
Table 2. Comparison of parameters between CC genotype and CT + TT genotype of 1908C/T polymorphism

\begin{tabular}{lccc}
\hline & CC & CT + TT & \\
& $(n=157)$ & $(n=104)$ & $p$ \\
\hline Age (years) & $65.3 \pm 0.9$ & $63.5 \pm 1.2$ & 0.24 \\
BMI $\left(\mathrm{kg} / \mathrm{m}^{2}\right)$ & $23.5 \pm 0.3$ & $23.7 \pm 0.3$ & 0.58 \\
SBP $(\mathrm{mmHg})$ & $133 \pm 1.7$ & $136 \pm 2.1$ & 0.30 \\
DBP $(\mathrm{mmHg})$ & $75 \pm 0.9$ & $77 \pm 1.1$ & 0.07 \\
Total cholesterol $(\mathrm{mg} / \mathrm{dL})$ & $194 \pm 2.7$ & $190 \pm 3.4$ & 0.09 \\
Triglyceride $(\mathrm{mg} / \mathrm{dL})$ & $112 \pm 5.1$ & $113 \pm 10.0$ & 0.91 \\
HDL cholesterol $(\mathrm{mg} / \mathrm{dL})$ & $52 \pm 0.9$ & $52 \pm 1.7$ & 0.96 \\
Current smoker $(\%)$ & 32.4 & 5.2 & 0.75 \\
HOMA-IR & $1.1 \pm 0.1$ & $1.0 \pm 0.2$ & 0.56 \\
hsCRP $(\mathrm{mg} / \mathrm{dL})$ & $0.11 \pm 0.008$ & $0.082 \pm 0.02$ & 0.10 \\
Adiponectin $(\mathrm{ng} / \mathrm{mL})$ & $6.1 \pm 0.2$ & $6.0 \pm 0.4$ & 0.80 \\
\hline
\end{tabular}

Values are expressed as the mean \pm SEM or \%. BMI, body mass index; SBP, systolic blood pressure; DBP, diastolic blood pressure; HDL, high-density lipoprotein; HOMA-IR, homeostasis assessment model of insulin resistance; hsCRP, highly sensitive C-reactive protein; baPWV, brachial-ankle pulse wave velocity

Table 3. Comparison of parameters between CC genotype and CT + TT genotype of -1030C/T polymorphism

\begin{tabular}{lccc}
\hline & CC & CT + TT & \\
& $(n=207)$ & $(n=54)$ & $p$ \\
\hline Age (years) & $64.8 \pm 0.8$ & $63.9 \pm 1.5$ & 0.61 \\
BMI (kg/m $\left.{ }^{2}\right)$ & $23.6 \pm 0.2$ & $23.2 \pm 0.4$ & 0.37 \\
SBP (mmHg) & $134 \pm 1.4$ & $131 \pm 2.8$ & 0.27 \\
DBP (mmHg) & $76 \pm 0.8$ & $74 \pm 1.6$ & 0.24 \\
Total cholesterol (mg/dL) & $191 \pm 2.2$ & $199 \pm 4.4$ & 0.38 \\
Triglyceride (mg/dL) & $112 \pm 5.9$ & $106 \pm 7.3$ & 0.56 \\
HDL cholesterol (mg/dL) & $53 \pm 1.0$ & $52 \pm 1.3$ & 0.88 \\
Current smoker $(\%)$ & 35.4 & 28.1 & 0.26 \\
HOMA-IR & $1.1 \pm 0.1$ & $1.0 \pm 0.1$ & 0.27 \\
hsCRP (mg/dL) & $0.11 \pm 0.01$ & $0.10 \pm 0.01$ & 0.43 \\
Adiponectin $(\mathrm{ng} / \mathrm{mL})$ & $6.0 \pm 0.3$ & $6.2 \pm 0.3$ & 0.52 \\
\hline
\end{tabular}

Values are expressed as the mean \pm SEM or \%. BMI, body mass index; SBP, systolic blood pressure; DBP, diastolic blood pressure; HDL, high-density lipoprotein; HOMA-IR, homeostasis assessment model of insulin resistance; hsCRP, highly sensitive C-reactive protein; baPWV, brachial-ankle pulse wave velocity

$\mathrm{C}$ allele (CC vs. CT+TT) for the two polymorphisms. Tables 2 and 3 show the clinical parameters of each genotype of the $1908 \mathrm{C} / \mathrm{T}$ polymorphism and $-1030 \mathrm{C} / \mathrm{T}$ polymorphism of $L M N A$, respectively. Despite previous findings ${ }^{6-8)}$, there was no significant relationship between the $\mathrm{T}$ allele of $1908 \mathrm{C} / \mathrm{T}$ polymorphism and metabolic traits in our study cohort. $\mathrm{BaPWV}$ of subjects with CC genotype of $-1030 \mathrm{C} / \mathrm{T}$ polymorphism was significantly greater than that of 
$1908 \mathrm{C} / \mathrm{T}$

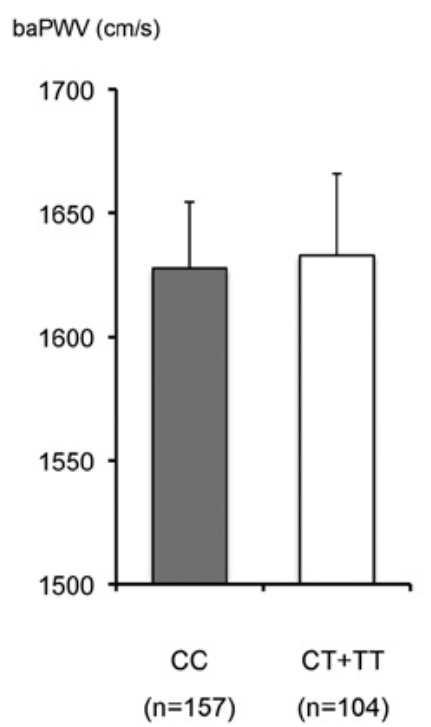

$-1030 \mathrm{C} / \mathrm{T}$

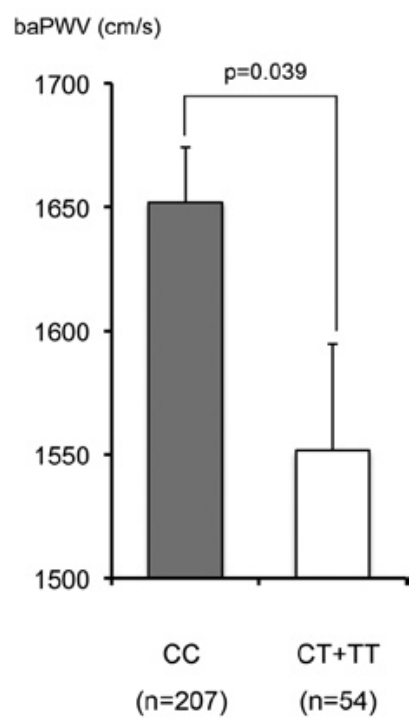

Fig. 1. Comparison of brachial-ankle pulse wave velocity (baPWV) according to genotypes of LMNA 1908C/T and $-1030 \mathrm{C} / \mathrm{T}$ polymorphisms.

subjects with CT + TT genotype of $-1030 \mathrm{C} / \mathrm{T}$ polymorphism $(1,652 \pm 22.1 \mathrm{~cm} / \mathrm{s}$ vs. $1,552 \pm 43.0 \mathrm{~cm} / \mathrm{s}$, $p=0.039)$, while there was no significant difference in the genotype of $1908 \mathrm{C} / \mathrm{T}$ polymorphism (Fig. 1). $\mathrm{BaPWV}$ showed significant positive correlations with age, systolic blood pressure and smoking habit and a significant negative correlation with body height. We therefore selected these factors as covariates of multiple regression analysis for baPWV and $L M N A$ $-1030 \mathrm{C} / \mathrm{T}$ polymorphism (Table 4). There was an independent relationship between the genotype of $-1030 \mathrm{C} / \mathrm{T}$ polymorphism and baPWV after adjusting covariates. Mean baPWVs of $-1030 / \mathrm{CC}$ and $-1030 / \mathrm{CT}$ + TT genotypes were $1,650 \pm 17.1 \mathrm{~cm} / \mathrm{s}$ and $1,571 \pm$ $32.0 \mathrm{~cm} / \mathrm{s}$, respectively, after adjusting covariates.

Since there was a strong linear correlation between age and baPWV, we analyzed the genotypic difference of $-1030 \mathrm{C} / \mathrm{T}$ in this correlation. The gradient of the regression line of subjects with CC genotype was significantly high compared to that of subjects with CT + TT genotype (data not shown), indicating that subjects with CC genotype of $-1030 \mathrm{C} / \mathrm{T}$ polymorphism might be susceptible to the progression of arterial stiffness by aging.

\section{Discussion}

We examined the hypothesis that there is a positive, independent association between polymorphisms
Table 4. Multiple regression analysis for brachial-ankle pulse wave velocity (baPWV)

\begin{tabular}{lcccc}
\hline Term & Estimate & SE & $t$ & $p$ \\
\hline Age & 12.5 & 1.58 & 7.9 & $<0.0001$ \\
Body height & 0.684 & 2.55 & 0.3 & 0.79 \\
Systolic blood pressure & 7.69 & 1.15 & 6.7 & $<0.0001$ \\
Smoking habit & -6.34 & 15.9 & -0.40 & 0.69 \\
-1030C/T & -39.4 & 17.8 & -2.2 & 0.028 \\
CC vs. CT + TT & & & & \\
\hline
\end{tabular}

$\mathrm{R}^{2}=0.48(n=261)$

of LMNA and arterial stiffness in a cross-sectional study of a Japanese population. Genotype frequencies of the two polymorphisms satisfied the Hardy-Weinberg equilibrium. The mean baPWV of $-1030 \mathrm{C} / \mathrm{T}$ polymorphism was significantly greater in subjects with CC genotype than in subjects with CT + TT genotype; however, no significant difference was found for $1908 \mathrm{C} / \mathrm{T}$ polymorphism. On the other hand, age, body height, systolic blood pressure, and smoking habit were significantly associated with baPWV. Multiple regression analysis including covariates revealed that subjects with the $-1030 \mathrm{~T}$ allele had a significantly lower level of baPWV.

The 1908C/T polymorphism of $L M N A$ has been reported to be related to metabolic abnormalities or insulin resistance in Japanese ${ }^{7)}$ and Armish ${ }^{8)}$, but a relationship between this polymorphism and arterial stiffness was not found in the present study. In addition, no relationship was found between 1908C/T polymorphism and metabolic traits in our population. This may due to the characteristics of our subjects. Subjects taking any medication were excluded from this study, and the number of subjects with type 2 diabetes mellitus or dyslipidemia was therefore small; however, the promoter $-1030 \mathrm{C} / \mathrm{T}$ polymorphism of $L M N A$, which we have recently identified by direct sequencing in the subjects, was independently associated with baPWV as a marker of arterial stiffness. To our knowledge, this is the first report of a relationship between $L M N A$ polymorphism and arterial stiffness.

In addition, we investigated the relation between the prevalence of cardiovascular diseases and SNPs in $L M N A$ using a cross-sectional method; however, there was no significant relation between cardiovascular diseases and SNPs. Because the subjects of our study were relatively healthy and had a low prevalence of cardiovascular diseases, our investigation might lack statistical power. A prospective study to elucidate this relation is now ongoing. Because of the shortness of the follow-up period, we do not have valuable results 
at present, but we will report the obtained results in the future.

Mutations in $L M N A$ have been discovered in a staggering variety of inherited diseases called "laminopathies ${ }^{20)}$ ". To date, several laminopathies are more familiar than Hutchinson-Gilford progeria syndrome (HGPS), such as Dunnigan-type familial partial lipodystrophy (FPLD), Emery-Dreifuss muscular dystrophy, Charcot-Marie-Tooth disease, limb-girdle muscular dystrophy, mandibuloacral dysplasia, dilated cardiomyopathy with conduction abnormality and early onset of atrial fibrillation. Laminopathies are caused by a mutation of $L M N A$, and are likely to include cardiovascular diseases ${ }^{21,22)}$. The precise mechanisms of this relationship are unknown, but it is speculated that $L M N A$ regulates metabolic traits as well as arterial stiffness and thus results in the aging process. HGPS is a laminopathy that is mainly caused by C-to-T mutation in position 1824 of LMNA and results in systemic arteriosclerosis. Recent studies have shown that this mutation causes nuclear blebbing induced by anchoring the mutant lamin A (called "progerin", which lacks 50 amino acids near the carboxy terminus) to the inner nuclear membrane, resulting in dysregulated gene transcription, heterochromatin disorganization $^{23,24)}$, and increased vulnerability of the nuclear membrane. Numerous abnormalities present in HGPS are common phenomena that occur in cells not only of HGPS patients but also aged individuals in the general population, such as nuclear blebbing, epigenetic changes and increased levels of DNA damage $^{25)}$; therefore, $L M N A$ seems to be one of the key genes regulating aging as well as arterial stiffness.

In order to elucidate the function of $-1030 \mathrm{C} / \mathrm{T}$ promoter polymorphism of $L M N A$, we searched for transcription factors likely to bind around $-1030 \mathrm{C} / \mathrm{T}$, using online databases of TRANSFAC, JASPAR, IMD, and CBIL/GibbsMat (http://www.cbil.upenn. edu/cgi-bin/tess/tess/). Motif analysis revealed one transcription factor, CREB-binding protein/CCAAT recognition factor (CBP/CRF), which has homology with the sequence including the $-1030 \mathrm{~T}$ allele. This may result in a difference in LMNA expression. Subjects with the $-1030 \mathrm{~T}$ allele are likely to have a strong expression of lamin $\mathrm{A}$ and $\mathrm{C}$ matrix and might have a stable nuclear membrane against environmental insult, represented by reactive oxygen species.

Our study has several limitations. First, our study was conducted using a small number of subjects in a Japanese population. Second, this study was is designed as a cross-sectional method. Although arteriosclerosis occurs mostly in aged individuals, the genotype-phenotype relationship showd also be analyzed in a time- considered, longitudinal study. Third, the mechanisms by which transcriptional activities of $L M N A$ are regulated by promoter $-1030 \mathrm{C} / \mathrm{T}$ polymorphism are unclear. Further study is required to clarify the function of promoter $-1030 \mathrm{C} / \mathrm{T}$ polymorphism.

In conclusion, promoter $-1030 \mathrm{C} / \mathrm{T}$ polymorphism of $L M N A$ might be associated with arterial stiffness in Japanese independent of metabolic traits. The mechanism of the influence of $L M N A$ on arterial stiffness may reveal part of the mechanism of a common condition susceptible to arteriosclerosis, aging.

\section{Acknowledgements}

We would like to express our gratitude to Ms. Kazuko Iwasa and Ms. Eriko Nagata for their continued support of our investigations. The present study was supported by a Grant-in-Aid for Scientific Research (H17-pharmaco-common-003) from the Japanese Ministry of Health, Labor, and Welfare, by Grants-in-Aid for Scientific Research (18590265, 18590811 and 19650188) from the Ministry of Education, Science, Sports and Culture of Japan, and by research grants from Takeda Science Foundation and the Japan Research Foundation for Clinical Pharmacology.

\section{References}

1) DeBusk FL: The Hutchinson-Gilford progeria syndrome. Report of 4 cases and review of the literature. J Pediatr, 1972; 80: 697-724

2) Hennekam RC: Hutchinson-Gilford progeria syndrome: review of the phenotype. Am J Med Genet A, 2006; 140: 2603-2624

3) Ogihara T, Hata T, Tanaka K, Fukuchi K, Tabuchi Y, Kumahara Y: Hutchinson-Gilford progeria syndrome in a 45-year-old man. Am J Med, 1986; 81: 135-138

4) Eriksson M, Brown WT, Gordon LB, Glynn MW, Singer J, Scott L, Erdos MR, Robbins CM, Moses TY, Berglund P, Dutra A, Pak E, Durkin S, Csoka AB, Boehnke M, Glover TW, Collins FS: Recurrent de novo point mutations in lamin A cause Hutchinson-Gilford progeria syndrome. Nature, 2003; 423: 293-298

5) Fukuchi K, Katsuya T, Sugimoto K, Kuremura M, Kim HD, Li L, Ogihara T: LMNA mutation in a 45 year old Japanese subject with Hutchinson-Gilford progeria syndrome. J Med Genet, 2004; 41: e67

6) Hegele RA, Huff MW, Young TK: Common genomic variation in LMNA modulates indexes of obesity in Inuit. J Clin Endocrinol Metab, 2001; 86: 2747-2751

7) Murase Y, Yagi K, Katsuda Y, Asano A, Koizumi J, Mabuchi $\mathrm{H}$ : An LMNA variant is associated with dyslipidemia and insulin resistance in the Japanese. Metabolism, 2002; 51: 1017-1021

8) Steinle NI, Kazlauskaite R, Imumorin IG, Hsueh WC, 
Pollin TI, O'Connell JR, Mitchell BD, Shuldiner AR: Variation in the lamin A/C gene: associations with metabolic syndrome. Arterioscler Thromb Vasc Biol, 2004; 24: 1708-1713

9) Weber MA: The measurement of arterial properties in hypertension. Am J Hypertens, 2001; 14: 183-185

10) Sato H, Hayashi J, Harashima K, Shimazu H, Kitamoto K: A population-based study of arterial stiffness index in relation to cardiovascular risk factors. J Atheroscler Thromb, 2005; 12: 175-180

11) Hatsuda $S$, Shoji T, Shinohara K, Kimoto E, Mori K, Fukumoto S, Koyama H, Emoto M, Nishizawa Y: Regional arterial stiffness associated with ischemic heart disease in type 2 diabetes mellitus. J Atheroscler Thromb, 2006; 13: $114-121$

12) Blacher J, Safar ME, Guerin AP, Pannier B, Marchais SJ, London GM: Aortic pulse wave velocity index and mortality in end-stage renal disease. Kidney Int, 2003; 63: 1852-1860

13) Boutouyrie P, Tropeano AI, Asmar R, Gautier I, Benetos A, Lacolley P, Laurent $S$ : Aortic stiffness is an independent predictor of primary coronary events in hypertensive patients: a longitudinal study. Hypertension, 2002; 39: $10-15$

14) Meaume S, Benetos A, Henry OF, Rudnichi A, Safar ME: Aortic pulse wave velocity predicts cardiovascular mortality in subjects $>70$ years of age. Arterioscler Thromb Vasc Biol, 2001; 21: 2046-2050

15) Ohnishi H, Saitoh S, Ura N, Takagi S, Obara F, Akasaka $\mathrm{H}$, Oimatsu H, Shimamoto K: Relationship between insulin resistance and accumulation of coronary risk factors. Diabetes Obes Metab, 2002; 4: 388-393

16) Ohnishi H, Saitoh $S$, Takagi $S$, Ohata J, Isobe T, Kikuchi Y, Takeuchi H, Shimamoto K: Pulse wave velocity as an indicator of atherosclerosis in impaired fasting glucose: the Tanno and Sobetsu study. Diabetes Care, 2003; 26: 437-440

17) Fujiwara T, Saitoh $S$, Takagi $S$, Ohnishi H, Ohata J, Takeuchi H, Isobe T, Chiba Y, Katoh N, Akasaka H, Shi- mamoto K: Prevalence of asymptomatic arteriosclerosis obliterans and its relationship with risk factors in inhabitants of rural communities in Japan: Tanno-Sobetsu study. Atherosclerosis, 2004; 177: 83-88

18) Takeuchi H, Saitoh S, Takagi S, Ohnishi H, Ohhata J, Isobe T, Shimamoto K: Metabolic syndrome and cardiac disease in Japanese men: applicability of the concept of metabolic syndrome defined by the National Cholesterol Education Program-Adult Treatment Panel III to Japanese men-the Tanno and Sobetsu Study. Hypertens Res, 2005; 28: 203-208

19) Munakata M, Ito N, Nunokawa T, Yoshinaga K: Utility of automated brachial ankle pulse wave velocity measurements in hypertensive patients. Am J Hypertens, 2003; 16: 653-657

20) Al-Shali KZ, Hegele RA: Laminopathies and atherosclerosis. Arterioscler Thromb Vasc Biol, 2004; 24: 1591-1595

21) Hegele RA: Monogenic forms of insulin resistance: apertures that expose the common metabolic syndrome. Trends Endocrinol Metab, 2003; 14: 371-377

22) Hegele RA, Kraw ME, Ban MR, Miskie BA, Huff MW, $\mathrm{Cao} \mathrm{H}$ : Elevated serum C-reactive protein and free fatty acids among nondiabetic carriers of missense mutations in the gene encoding lamin A/C (LMNA) with partial lipodystrophy. Arterioscler Thromb Vasc Biol, 2003; 23: 111116

23) Capell BC, Collins FS, Nabel EG: Mechanisms of cardiovascular disease in accelerated aging syndromes. Circ Res, 2007; 101: 13-26

24) Merideth MA, Gordon LB, Clauss S, Sachdev V, Smith AC, Perry MB, Brewer CC, Zalewski C, Kim HJ, Solomon B, Brooks BP, Gerber LH, Turner ML, Domingo DL, Hart TC, Graf J, Reynolds JC, Gropman A, Yanovski JA, Gerhard-Herman M, Collins FS, Nabel EG, Cannon RO 3rd, Gahl WA, Introne WJ: Phenotype and course of Hutchinson-Gilford progeria syndrome. N Engl J Med, 2008; 358: 592-604

25) Scaffidi P, Misteli T: Lamin A-dependent nuclear defects in human aging. Science, 2006; 312: 1059-1063 